\title{
Formação em Saúde da Família e sua associação com processos de trabalho das Equipes de Saúde Bucal da Atenção Básica
}

\author{
Training in Family Health and its association with work processes \\ of Primary Care Dentistry Teams
}

Nathália Maria Lopes dos Santos ${ }^{1}$

Fernando Neves Hugo ${ }^{2}$

${ }^{1}$ Programa de PósGraduação em Odontologia, Faculdade de Odontologia, Universidade Federal do Rio Grande do Sul (UFRGS). R. Ramiro Barcelos 2492, Rio Branco. 90030-003 Porto Alegre RS Brasil. nathaliamls@terra.com.br ${ }^{2}$ Departamento de Odontologia Preventiva e Social, Faculdade de Odontologia, UFRGS. Porto Alegre RS Brasil.

\begin{abstract}
The National Oral Health Policy emphasizes the importance of training in Primary Health Care and adapting the work processes to the new health care model. This study seeks to investigate the association between the training of Dental-Surgeons in Family Health and the work process. It involved a cross-sectional and multicentric study, with 18,114 Oral Health Teams that participated on the National Program to Enhance Access and Quality of Primary Health Care. Data were collected through interviews, using a structured questionnaire. Professionals with post-graduate education in Family Health share their schedule with other Primary Care professionals more frequently (PR: 1.04; CI 95\%: 1.03 - 1.06), organize their schedule to offer educational activities in Oral Health more frequently (PR:1.03; CI 95\%: 1.02 - 1.04), ensure completion of the treatment of patients more frequently (OR: 1.68; CI 95\%: 1.19-2.38), conduct more home visits (PR: 1.08; CI 95\%: 1.07 - 1.09) and use more protocols for prioritizing actions more frequently (PR: 1.06; CI 95\%:1.04 - 1.07). Investment in training in Family Health may be important for better performance in Primary Care Dentistry, thereby leading to changes in the model of Comprehensive Oral Health care.
\end{abstract}

Key words Professional training, Oral health, Primary health care, Health evaluation, Family health
Resumo A Política Nacional de Saúde Bucal aponta como pressuposto qualificar a Atenção Básica e adequar o processo de trabalho ao novo modelo de atenção à saúde. Objetiva-se investigar a associação entre a formação profissional dos Cirurgiões-Dentistas em Saúde da Família e o processo de trabalho. Estudo transversal, multicêntrico, com 18.114 Equipes de Saúde Bucal que aderiram ao segundo ciclo do Programa Nacional de Melhoria do Acesso e da Qualidade da Atenção Básica. Os dados foram coletados por meio de entrevistas, através de instrumento estruturado. Profissionais com formação em Saúde da Família compartilham mais sua agenda com os outros profissionais da Atenção Básica (RP: 1,04; IC de 95\%: 1,03 - 1,06), organizam mais a agenda para ofertar atividades educativas de Saúde Bucal (RP: 1,03; IC de 95\%: 1,02 - 1,04), garantem conclusão de tratamento dos usuários mais frequentemente (OR: 1,68; IC de 95\%: 1,19-2,38), realizam mais visitas domiciliares (RP: 1,08; IC de 95\%: 1,07 1,09) e utilizam mais protocolos para definição de ações prioritárias (RP: 1,06; IC de 95\%:1,041,07). Investir em formação em Saúde da Família pode ser importante, portanto, para a efetivação de mudanças no modelo de atenção à Saúde Bucal, o qual ainda é pouco preocupado no cuidado integral.

Palavras-chave Formação profissional, Saúde bucal, Atenção básica, Avaliação em saúde, Saúde da família 


\section{Introdução}

No Brasil, a consolidação da avaliação começa lentamente a ter contornos mais nítidos e a se tornar prática institucional e institucionalizada ${ }^{1}$. A partir dos anos 2000, observaram-se esforços no sentido de avaliar a Atenção Básica (AB) em Saúde em seus diversos aspectos, empreendidos por centros acadêmicos e fortemente induzidos pelo Ministério da Saúde (MS) por meio de financiamento, apoio e realização de pesquisas na área de monitoramento e avaliação ${ }^{2}$. A Política Nacional de Saúde Bucal (PNSB), em 2004, aponta como pressuposto importante assumir o compromisso de qualificação da $\mathrm{AB}$, bem como ampliar e qualificar a assistência em Saúde Bucal (SB) para reorientar e adequar o processo de trabalho ao novo modelo de atenção à saúde proposto ${ }^{3}$. Assim, no contexto do conjunto de ações e programas que fazem parte da nova Política Nacional de Atenção Básica (PNAB), o MS lançou, em 2011, o Programa Nacional de Melhoria do Acesso e da Qualidade da Atenção Básica (PMAQ-AB), como principal estratégia indutora de mudanças nas condições e modos de funcionamento das Unidades Básicas de Saúde (UBS) ${ }^{4,5}$.

A partir do PMAQ, aposta-se, assim, na produção de uma cultura de análise, avaliação e intervenção capaz de gerar capacidade institucional nas equipes e nos sistemas locais para produzir mudanças nas práticas dos serviços, visto que há necessidade de qualificação dos processos de trabalho das Equipes de Atenção Básica (EAB), incluindo as Equipes de Saúde Bucal (ESB). Para isso, avalia, reconhece e valoriza, através de incentivos financeiros, aqueles que avançam na direção dos resultados esperados, segundo padrões de qualidade estabelecidos nacionalmente ${ }^{4}$.

Para reafirmar o papel da $\mathrm{AB}$ como porta de entrada preferencial dos serviços de saúde e estimular a real mudança no modelo de atenção alicerçado pela Estratégia de Saúde da Família (ESF), há a necessidade de se investir continuamente na formação e qualificação dos profissionais de saúde para o trabalho no Sistema Único de Saúde (SUS). O desenvolvimento de recursos humanos, desta forma, representa um papel de suma importância para a consecução de um SUS democrático, equitativo e eficiente ${ }^{6}$. Pensando nisso, o MS, desde o fim da década de 90 , vem investindo na capacitação de recursos humanos para a $\mathrm{AB}$, através de cursos de aperfeiçoamento, de atualização e de pós-graduação lato sensu nas modalidades Especialização e Residência Multiprofissional em Saúde da Família $(\mathrm{SF})^{7}$.
Profissionais com formação voltada para o SUS tendem a conhecê-lo melhor, assim como as políticas de saúde, adquirindo uma postura de atuação compromissada com as necessidades do mesmo ${ }^{8,9}$, importante para o seu efetivo engajamento nas propostas de transformação das práticas profissionais e da própria organização do trabalho ${ }^{8}$. São capazes de prestar uma atenção integral mais humanizada, de trabalhar em equipe, de compreender melhor a realidade em que vive a população, rompendo com o modelo flexneriano de formação que privilegia a abordagem individual e curativa, é pautado nas especialidades, fragmentado, incapaz de transcender o espaço do consultório e propor um diagnóstico sobre o coletivo e suas intervenções ${ }^{10}$. Entretanto, não há estudos abrangentes, de base nacional e com validade externa que evidenciem se a formação em saúde da família está associada a melhor desempenho em processos de trabalho que são estratégicos para a reorientação da atenção proposta tanto pela PNAB como pela PNSB.

A formação do Cirurgião-Dentista $(C D)$ em $\mathrm{SF}$, assim como outros profissionais da EAB, deve estar pautada na capacidade de planejamento, organização, desenvolvimento e avaliação das ações que respondam às necessidades da comunidade $^{11}$, para além do paradigma curativo e pontual. Além disso, conforme a PNSB, os profissionais da SB devem: atuar interdisciplinarmente e multidisciplinarmente, não se limitando ao campo biológico ou técnico-odontológico, mas sim interagindo com profissionais de outras áreas; assegurar integralidade de atenção em SB, articulando o individual com o coletivo, a promoção e a prevenção com o tratamento e a recuperação da saúde da população adscrita; utilizar a epidemiologia e as informações sobre o território subsidiando o planejamento das ações; centrar a atuação na vigilância à saúde, incorporando práticas contínuas de avaliação e acompanhamento dos danos, riscos e determinantes do processo saúde-doença; e ampliar e qualificar a assistência prestada, considerando acesso, acolhimento, vínculo, ética e responsabilidade profissional ${ }^{3}$. Ter formação profissional adequada para trabalhar na $A B$ é, portanto, fundamental para o aperfeiçoamento dos serviços de saúde e para as relações sociais estabelecidas inter e intra-equipes e com os usuários, proporcionando uma melhora na qualidade da assistência prestada ${ }^{11}$.

Logo, devido à falta de evidências na área de avaliação da formação profissional dos CD das ESB e sua relação com os processos de trabalho por eles desenvolvidos, este estudo tem como 
objetivo investigar a associação entre a formação profissional dos CD na área de SF e os indicadores de processo de trabalho que são desenvolvidos pelas ESB da $\mathrm{AB}$, levando em consideração a organização da demanda programática e a realização de visitas domiciliares (VD).

\section{Métodos}

Este é um estudo observacional transversal realizado a partir dos dados do segundo ciclo do PMAQ-AB. Possui abrangência nacional e foi executado de forma multicêntrica e integrada, por diversas Instituições de Ensino e Pesquisa e com o acompanhamento direto do MS. Abrangeu 5.070 municípios brasileiros (91\%), 30.424 EAB (90\%) e 19.898 ESB (89\%) em todas as regiões do Brasil ${ }^{4}$. Todas as EAB, incluindo as de Saúde Bucal (SB), puderam aderir ao PMAQ-AB voluntariamente, pressupondo um processo inicial de pactuação entre equipes e gestores municipais. A adesão se deu por meio de formulário eletrônico preenchido pelos gestores municipais, disponibilizado no sítio do programa, sendo avaliadas apenas as equipes que formalizaram a adesão.

As informações deste estudo são referentes à etapa de Avaliação Externa do Programa, sobre as avaliações do Módulo VI, respondidas pelos profissionais de SB nas Unidades de Saúde ( $\mathrm{n}=$ 18.114), mediante entrevista e verificação de documentações in loco. As avaliações foram realizadas com contato prévio do entrevistador com o profissional respondente, sendo este último, na maioria das vezes, o CD.

A Avaliação Externa iniciou-se após a fase de adesão dos municípios ao Programa, sendo realizada entre novembro de 2013 e julho de 2014. Para o trabalho em campo, foram selecionados avaliadores externos que participaram de um processo de formação centrado nas atividades que posteriormente desenvolveriam. Os avaliadores foram treinados uniformemente por meio da utilização de um manual de campo elaborado pelo Departamento de Atenção Básica (DAB) e pelas Instituições de Ensino e Pesquisa parceiras do PMAQ -AB. A coleta foi realizada por meio da utilização de computadores portáteis do tipo tablet, os quais continham um aplicativo com o instrumento padronizado e testado previamente, abrangendo sete módulos com questões que avaliaram aspectos referentes à estrutura das UBS, aos processos de trabalho desenvolvidos pelas ESB, entre outros.

Após a realização da Avaliação Externa, os dados coletados foram enviados via rede a um servidor do MS para que fossem validados. Para o estabelecimento de um padrão mínimo e máximo na averiguação da informação coletada in loco, os parâmetros de validação, que estão disponíveis no manual intitulado "Protocolo de análise da consistência e validação dos dados coletados", distribuído pelo MS às Universidades que realizaram a avaliação externa do programa, são os seguintes: a) tempo de coleta (no módulo II entre 1 e 2 horas e, ainda, devido à complexidade e à natureza detalhada dos questionários, foi definido que cada bloco dentro do módulo deveria durar no mínimo 15 minutos). Uma aplicação menor poderia indicar que o avaliador não aplicou devidamente as perguntas elencadas em cada bloco. O início e o término da aplicação deveriam ser entre as 7 e 19 horas. b) Proporção de resposta "Não sabe/Não Respondeu” (não podendo passar de $15 \%$ do total de respostas de cada bloco). c) Correspondência das coordenadas geográficas captadas pelo tablet e a localização da UBS. Caso alguns desses parâmetros não estivesse dentro dos padrões definidos pelo MS, o supervisor de campo deveria entrar em um ambiente eletrônico virtual específico de validação das informações e justificar essa inconsistência.

Foram selecionadas variáveis do instrumento de Avaliação Externa do PMAQ-AB referentes à ESB que pudessem refletir questões relacionadas à formação e qualificação dos profissionais de SB e ao processo de trabalho das mesmas, no que se refere à organização da demanda programática e realização de visitas domiciliares.

As variáveis foram descritas no instrumento da Avaliação Externa do PMAQ-AB, módulo VI, "Entrevista com Profissional da Equipe de Saúde Bucal e Verificação de Documentos na Unidade

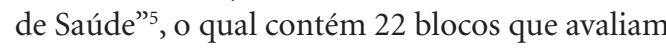
informações relacionadas ao processo de trabalho das ESB. Foram selecionados os seguintes indicadores, conforme quadro abaixo (Quadro 1):

Além destas variáveis apresentadas acima, foram analisadas no presente estudo as macrorregiões (Norte/Nordeste/Centro-Oeste/Sul/Sudeste) onde cada equipe estava localizada, para fins de ajuste somente de potencial de confundimento.

No sentido de possibilitar uma melhor análise da relação entre formação profissional (graduação e formação em SF) e processo de trabalho das ESB, algumas variáveis foram criadas e outras recategorizadas. "Formação em SF - com opção de resposta Especialização e Residência? (a. sim; b. não)" foi criada; "Qual o seu tipo de vínculo?", com 8 opções de respostas, foi recategorizada em 4 respostas - “a. Estatutário; b. CLT (o qual 
Quadro 1. Indicadores avaliados no PMAQ-AB.

\begin{tabular}{|c|c|}
\hline Variáveis & Opções de resposta \\
\hline Possui ou está em formação complementar? & $\begin{array}{l}\text { Sim } \\
\text { Não }\end{array}$ \\
\hline Possui quais destes processos de formação? & $\begin{array}{l}\text { Especialização em SF } \\
\text { Residência em SF }\end{array}$ \\
\hline Qual é o seu tipo de vínculo? & $\begin{array}{l}\text { Servidor público estatutário } \\
\text { Cargo comissionado } \\
\text { Contrato temporário pela administração pública regido } \\
\text { por legislação especial (municipal/estadual/federal) } \\
\text { Contrato temporário por prestação de serviço } \\
\text { Emprego público CLT } \\
\text { Contrato CLT } \\
\text { Autônomo } \\
\text { Outros } \\
\text { Não sabe ou não respondeu }\end{array}$ \\
\hline A agenda de atendimento clínico da equipe garante: & $\begin{array}{l}\text { Apenas consultas odontológicas agendadas } \\
\text { Apenas consultas odontológicas de demanda espontânea } \\
\text { Consultas odontológicas espontâneas e de agenda }\end{array}$ \\
\hline $\begin{array}{l}\text { Do total de pacientes atendidos, por semana, } \\
\text { quantos são agendados? }\end{array}$ & $\begin{array}{l}\text { Todos } \\
\text { Maior parte, os demais são de demanda espontânea } \\
\text { Metade, os demais são de demanda espontânea } \\
\text { Nenhum, todos são de demanda espontânea }\end{array}$ \\
\hline $\begin{array}{l}\text { A agenda da ESB é compartilhada com os } \\
\text { profissionais da Equipe da Saúde da Família? }\end{array}$ & $\begin{array}{l}\text { Sim } \\
\text { Não }\end{array}$ \\
\hline $\begin{array}{l}\text { A agenda da ESB está organizada para ofertar } \\
\text { atividades educativas de SB no território? }\end{array}$ & $\begin{array}{l}\text { Sim } \\
\text { Não }\end{array}$ \\
\hline $\begin{array}{l}\text { Como são agendadas as consultas odontológicas na } \\
\text { Unidade de Saúde? }\end{array}$ & $\begin{array}{l}\text { Em qualquer dia da semana, em qualquer horário } \\
\text { Em qualquer dia da semana, em horários específicos } \\
\text { Dias específicos fixos em até } 3 \text { dias na semana } \\
\text { Dias específicos fixos em mais de } 3 \text { dias na semana } \\
\text { Dia específico fixo uma vez por mês }\end{array}$ \\
\hline $\begin{array}{l}\text { A ESB garante agendamento de retorno para a } \\
\text { continuidade do tratamento de um usuário que } \\
\text { iniciou seu tratamento? }\end{array}$ & $\begin{array}{l}\text { Sim } \\
\text { Não }\end{array}$ \\
\hline A ESB realiza tratamento concluído (TC)? & $\begin{array}{l}\text { Para todos os usuários atendidos } \\
\text { Para alguns usuários atendidos } \\
\text { Nunca realiza TC }\end{array}$ \\
\hline A ESB realiza visita domiciliar? & $\begin{array}{l}\text { Sim } \\
\text { Não }\end{array}$ \\
\hline $\begin{array}{l}\text { A ESB utiliza algum protocolo ou documento com } \\
\text { definição de situações prioritárias para a visita } \\
\text { domiciliar? }\end{array}$ & $\begin{array}{l}\text { Sim } \\
\text { Não }\end{array}$ \\
\hline
\end{tabular}

contempla as opções da entrevista referentes a emprego público CLT e contrato CLT); c. Contrato temporário ( o qual contempla as opções da entrevista referentes a cargo comissionado, contrato temporário pela administração pública regido por legislação especial (municipal/estadual/ federal) e contrato temporário por prestação de serviço) e d. Outros (o qual contempla as opções da entrevista referentes a autônomo, outros e não sabe ou não respondeu)"; Como são agendadas as consultas odontológicas na US?" Com 5 opções de resposta, foi recategorizada em 2 possíveis: a. em qualquer dia da semana, em qualquer horário; b. em dias e horários específicos ( a qual contempla as opções da entrevista referentes a qualquer dia da semana, em horários específicos; 
dias específicos fixos em até 3 dias na semana; dias específicos fixos em mais de 3 dias na semana; dia específico fixo uma vez por mês).

Os dados foram analisados no software SPSS v18. Foram realizadas análises das frequências das variáveis, realizando-se o teste de qui-quadrado de Pearson para avaliar diferenças significativas ( $p<0,05)$, segundo formação profissional, para os desfechos de interesse. Foram realizadas análises multivariadas para verificar se havia associação entre a formação e os indicadores de processo de trabalho, por meio de Regressão de Poisson com variância robusta no caso de desfechos categóricos dicotômicos, e da Regressão Multinomial no caso de desfechos politômicos. São apresentadas Razões de Prevalência, quando da análise de desfechos dicotômicos, e Odds Ratios, no caso de desfechos politômicos, com seus respectivos intervalos de $95 \%$ de confiança. Foi utilizado o nível de significância de $5 \%$.

Este estudo, registrado sob o número 21904, foi submetido à análise do Comitê de Ética da Universidade Federal do Rio Grande do Sul e aprovado em 01 de março de 2012, por estar adequado ética e metodologicamente, de acordo com a resolução 196/96 e resoluções complementares do Conselho Nacional de Saúde (CNS).

\section{Resultados}

Os dados demonstram que, das 19.898 ESB que aderiram ao segundo ciclo do PMAQ, 18.114 ESB participaram da Avaliação Externa. Como pode ser visto na Tabela 1 , há um maior número de equipes pertencentes à macrorregião Nordeste $(42,5 \%)$. Quanto à formação complementar, 12.581 dos CD respondentes $(69,5 \%)$ possuem algum tipo, sendo destes 4.272 (34\%) em SF (Especialização ou Residência). No que se refere ao vínculo, 17.956 responderam, sendo que 7.993 $(44,5 \%)$ são estatutários. Quanto ao processo de trabalho, 16.304 (90\%) fornecem consultas agendadas e de demanda espontânea, 13.373 $(73,8 \%)$ agendam a maior parte dos pacientes, $9.500(52,4 \%)$ permitem o agendamento das consultas em dias e horários específicos, 12.007 $(66,3 \%)$ compartilham a agenda com a EAB, 16.407 (90,6\%) organizam a agenda para ofertar atividades de educação em SB no território. Além disso, 16.639 (91,9\%) garantem retorno para continuidade de tratamento de quem já iniciou e $11.224(62 \%)$ realizam tratamento concluído para todos os usuários. Quanto às visitas domiciliares, $14.178(78,3 \%)$ das ESB respondentes as realizam, mas somente $7.455(41,2 \%)$ utilizam protocolo para definiçãa de ações prioritárias para a realização de VD, enquanto $3936(21,7 \%)$ não souberam responder se há a utilização de algum protocolo.

Na Tabela 2, pode-se constatar a distribuição das variáveis segundo formação profissional e os resultados do teste qui-quadrado de Pearson. Todas as associações, no que se refere ao processo de trabalho desenvolvido pelas ESB da AB e a formação em SF na modalidade Especialização e Residência, foram estatisticamente significantes.

De acordo com o modelo de Regressão Multinomial, profissionais sem formação em SF apresentaram chance 1,68 vezes maior (IC 95\%: 1,19 a 2,38 ) de nunca realizar tratamento concluído (Tabela 3).

Conforme Regressão de Poisson (Tabela 4), profissionais sem formação em SF apresentam prevalência $4 \%$ maior de não compartilhamento da agenda com a EAB e $3 \%$ maior de não organização da agenda para ofertar atividades de educação em SB no território, em comparação aos profissionais com formação em SF. Com relação às $\mathrm{VD}$, profissionais sem formação em SF possuem prevalência $8 \%$ maior de não realização de VD, bem como $6 \%$ maior de não utilização de protocolo para definição de ações prioritárias para VD.

\section{Discussão}

Os achados deste trabalho são pioneiros ao abordar a influência da formação profissional nos processos de trabalhos desenvolvidos pelas ESB no Brasil. Além disso, referem-se quase que a totalidade das ESB do Brasil (81\%) e, portanto, representam o modelo de atenção à SB predominante no país, segundo a formação dos profissionais que dela participam. Indicam que, apesar de somente 34\% (4.272) dos profissionais terem formação em SF, houve associação significativa entre esta formação e melhor desempenho em processos de trabalho mais adequados às premissas do SUS de acesso, trabalho em equipe, integralidade e longitudinalidade. No que tange as variáveis Macrorregião e Tipo de Vínculo, estas não tiveram associação estatisticamente significativa com a exposição principal, visto que há profissionais em todas as regiões brasileiras com Especialização e/ou Residência em SF, assim como nos vínculos trabalhistas relatados. $\mathrm{O}$ que há diverso, assim, é a polarização dos profissionais pós-graduados e a maior flexibilização de vínculos trabalhistas para os municípios de 
Tabela 1. Frequências das variáveis do estudo - Avaliação Externa PMAQ 2013/2014.

\begin{tabular}{|c|c|c|}
\hline \multicolumn{2}{|c|}{ Variável } & \multirow{2}{*}{$\frac{\mathbf{n}(\%)}{1263(7,0 \%)}$} \\
\hline Macrorregião & Norte & \\
\hline & Nordeste & $7700(42,5 \%)$ \\
\hline & Centro-Oeste & $1572(8,7 \%)$ \\
\hline & Sul & $2552(14,1 \%)$ \\
\hline & Sudeste & $5027(27,8 \%)$ \\
\hline \multirow[t]{2}{*}{ Formação complementar } & Sim & $12581(69,5 \%)$ \\
\hline & Não & $5533(30,5 \%)$ \\
\hline \multirow[t]{2}{*}{ Formação complementar em SF } & Sim & $4272(34 \%)$ \\
\hline & Não & $8309(66,0 \%)$ \\
\hline \multirow[t]{4}{*}{ Tipo de vínculo } & Estatutário & $7993(44,5 \%)$ \\
\hline & CLT & $6732(16,7 \%)$ \\
\hline & Contrato temporário & $2997(37,5 \%)$ \\
\hline & Outros & $234(1,3 \%)$ \\
\hline \multirow[t]{3}{*}{ Tipos de consultas garantidas } & Apenas agendadas & $543(3,0 \%)$ \\
\hline & Apenas de demanda espontânea & $1267(7,0 \%)$ \\
\hline & Agendadas e de demanda espontânea & $16304(90,0 \%)$ \\
\hline \multirow{4}{*}{$\begin{array}{l}\text { Número de agendados do total de } \\
\text { pacientes }\end{array}$} & Todos & $891(4,9 \%)$ \\
\hline & Maior parte & $13373(73,8 \%)$ \\
\hline & Metade & $3054(16,9 \%)$ \\
\hline & Nenhum & $796(4,4 \%)$ \\
\hline \multirow[t]{2}{*}{ Forma de agendamento das consultas } & Em qualquer dia, em qualquer horário & $8614(47,6 \%)$ \\
\hline & Em dias e horários específicos & $9500(52,4 \%)$ \\
\hline \multirow[t]{2}{*}{ Agenda compartilhada com a EAB } & Sim & $12007(66,3 \%)$ \\
\hline & Não & $6107(33,7 \%)$ \\
\hline \multirow{2}{*}{$\begin{array}{l}\text { Agenda organizada para ofertar atividades } \\
\text { de educação em SB no território }\end{array}$} & Sim & $16407(90,6 \%)$ \\
\hline & Não & $1707(9,4 \%)$ \\
\hline \multirow{2}{*}{$\begin{array}{l}\text { Garantia de retorno para continuidade de } \\
\text { tratamento }\end{array}$} & Sim & $16639(91,9 \%)$ \\
\hline & Não & $1475(8,1 \%)$ \\
\hline \multirow[t]{3}{*}{ Realização de tratamento concluído } & Todos usuários & $11224(62,0 \%)$ \\
\hline & Alguns usuários & $6500(35,9 \%)$ \\
\hline & Nunca Realiza & $390(2,2 \%)$ \\
\hline \multirow[t]{2}{*}{ Realização de Visitas Domiciliares } & Sim & $14178(78,3 \%)$ \\
\hline & Não & $3936(21,7 \%)$ \\
\hline \multirow{2}{*}{$\begin{array}{l}\text { Utilização de protocolo para definição de } \\
\text { ações prioritárias para VD }\end{array}$} & Sim & $6723(47,4 \%)$ \\
\hline & Não & $7455(52,6 \%)$ \\
\hline
\end{tabular}

maior porte populacional, de acordo com Seidl et al. ${ }^{12}$. Quanto aos aspectos relacionados à demanda programática, profissionais com formação em SF compartilham mais sua agenda com os demais profissionais da EAB, organizam mais a agenda para ofertar atividades educativas de saúde bucal no território, garantem mais o retorno daqueles usuários que já iniciaram tratamento e garantem conclusão de tratamento dos usuários mais frequentemente. Além disso, no que se refere às VD, realizam mais VD e utilizam mais protocolos para definição de ações prioritárias nas VD.
Isto reafirma a importância de formar profissionais voltados para a SF e para o SUS, visto que esta formação apresenta-se como alternativa para promover a mudança da prática assistencial em saúde, sendo capaz de favorecer o trabalho em equipe, as trocas efetivas de saberes e práticas e a construção de uma nova realidade de saúde para a população. Tem como objetivo direcionar a atuação dos profissionais para atividades clínico-assistenciais aplicadas ao cuidado direto aos indivíduos e famílias em seu ambiente familiar e social ${ }^{6}$. Assim, é essencial que o investimento 
Tabela 2. Distribuição das variáveis estudadas segundo formação profissional.

\begin{tabular}{|c|c|c|c|c|}
\hline \multirow[b]{2}{*}{ Variável } & & \multicolumn{3}{|c|}{ Formação profissional } \\
\hline & & Graduação & $\begin{array}{c}\text { Especialização e Residência } \\
\text { em Saúde da Família }\end{array}$ & $\begin{array}{c}\mathbf{P} \\
\left(\mathbf{x}^{2}\right)\end{array}$ \\
\hline \multirow{3}{*}{$\begin{array}{l}\text { Tipos de consultas } \\
\text { garantidas }\end{array}$} & Apenas agendadas & $220(65,3 \%)$ & $117(34,7 \%)$ & 0,02 \\
\hline & $\begin{array}{l}\text { Apenas de demanda } \\
\text { espontânea }\end{array}$ & $566(70,6 \%)$ & $236(29,4 \%)$ & \\
\hline & $\begin{array}{l}\text { Agendadas e de demanda } \\
\text { espontânea }\end{array}$ & $7523(65,7 \%)$ & $3919(34,3 \%)$ & \\
\hline \multirow{4}{*}{$\begin{array}{l}\text { Número de agendados do } \\
\text { total de pacientes }\end{array}$} & Todos & $373(68,1 \%)$ & $175(31,9 \%)$ & 0,01 \\
\hline & Maior parte & $6143(65,1 \%)$ & $3297(34,9 \%)$ & \\
\hline & Metade & $1434(68,0 \%)$ & $675(32,0 \%)$ & \\
\hline & Nenhum & $359(74,2 \%)$ & $125(25,8 \%)$ & \\
\hline \multirow[t]{2}{*}{$\begin{array}{l}\text { Forma de agendamento } \\
\text { das consultas }\end{array}$} & $\begin{array}{l}\text { Em qualquer dia, em qualquer } \\
\text { horário }\end{array}$ & $3764(64,1 \%)$ & $2102(35,9 \%)$ & 0,01 \\
\hline & Em dias e horários específicos & $4545(67,7 \%)$ & $2168(32,3 \%)$ & \\
\hline \multirow{2}{*}{$\begin{array}{l}\text { Agenda compartilhada } \\
\text { com a EAB }\end{array}$} & Sim & $5557(64,9 \%)$ & $3008(35,1 \%)$ & 0,01 \\
\hline & Não & $2752(68,5 \%)$ & $1264(31,5 \%)$ & \\
\hline \multirow{2}{*}{$\begin{array}{l}\text { Agenda organizada } \\
\text { para ofertar atividades } \\
\text { de educação em SB no } \\
\text { território }\end{array}$} & Sim & $7550(65,4 \%)$ & $4000(34,6 \%)$ & 0,01 \\
\hline & Não & $759(73,6 \%)$ & $272(26,4 \%)$ & \\
\hline \multirow{2}{*}{$\begin{array}{l}\text { Garantia de retorno } \\
\text { para continuidade de } \\
\text { tratamento }\end{array}$} & Sim & $7634(65,7 \%)$ & $3985(34,3 \%)$ & 0,01 \\
\hline & Não & $675(70,2 \%)$ & $287(29,8 \%)$ & \\
\hline \multirow{3}{*}{$\begin{array}{l}\text { Realização de tratamento } \\
\text { concluído }\end{array}$} & Todos usuários & $5058(64,6 \%)$ & $2777(35,4 \%)$ & 0,01 \\
\hline & Alguns usuários & $3068(67,9 \%)$ & $1452(32,1 \%)$ & \\
\hline & Nunca realiza & $183(81,0 \%)$ & $43(19,0 \%)$ & \\
\hline \multirow{2}{*}{$\begin{array}{l}\text { Realização de Visitas } \\
\text { Domiciliares }\end{array}$} & Sim & $6474(63,4 \%)$ & $3735(36,6 \%)$ & 0,01 \\
\hline & Não & $1835(77,4 \%)$ & $537(22,6 \%)$ & \\
\hline \multirow{2}{*}{$\begin{array}{l}\text { Utilização de protocolo } \\
\text { para definição de ações } \\
\text { prioritárias para VD }\end{array}$} & Sim & $2964(58,8 \%)$ & $2080(41,2 \%)$ & 0,01 \\
\hline & Não & $3510(68,0 \%)$ & $1655(32,0 \%)$ & \\
\hline
\end{tabular}

Tabela 3. Regressão Multinomial segundo formação profissional, tendo como referência Especialização e Residência em Saúde da Família.

\begin{tabular}{|c|c|c|c|c|c|}
\hline \multirow{2}{*}{ Variável } & & \multicolumn{4}{|c|}{ Graduação } \\
\hline & & OR bruto (IC 95\%) & p & OR ajustado (IC 95\%) & $\mathbf{p}$ \\
\hline \multirow{3}{*}{$\begin{array}{l}\text { Tipos de } \\
\text { consultas } \\
\text { garantidas }\end{array}$} & Apenas agendadas & $0,98(0,78-1,23)$ & 0,86 & $0,97(0,76-1,23)$ & 0,79 \\
\hline & Apenas de demanda espontânea & $1,25(1,07-1,46)$ & 0,01 & $1,13(0,96-1,33)$ & 0,15 \\
\hline & Agendadas e de demanda espontânea & 1 & & 1 & \\
\hline \multirow{4}{*}{$\begin{array}{l}\text { Número de } \\
\text { agendados } \\
\text { do total de } \\
\text { pacientes }\end{array}$} & Todos & 1 & & 1 & \\
\hline & Maior parte & $0,87(0,73-1,05)$ & 0,15 & $0,90(0,74-1,09)$ & 0,30 \\
\hline & Metade & $1,00(0,81-1,22)$ & 0,97 & $0,92(0,74-1,09)$ & 0,46 \\
\hline & Nenhum & $1,35(1,03-1,77)$ & 0,03 & $1,08(0,82-1,44)$ & 0,57 \\
\hline \multirow{3}{*}{$\begin{array}{l}\text { Realização } \\
\text { de } \\
\text { tratamento } \\
\text { concluído }\end{array}$} & Todos usuários & 1 & & 1 & \\
\hline & Alguns usuários & $1,16(1,07-1,25)$ & 0,01 & $1,00(0,92-1,09)$ & 0,95 \\
\hline & Nunca realiza & $2,34(1,67-3,27)$ & 0,01 & $1,68(1,19-2,38)$ & 0,01 \\
\hline
\end{tabular}

${ }^{*}$ Ajustada para Macrorregião. 
Tabela 4. Regressão de Poisson segundo formação profissional, tendo como referência Especialização e Residência em Saúde da Família.

\begin{tabular}{|c|c|c|c|c|}
\hline \multirow[b]{2}{*}{ Variável } & \multicolumn{4}{|c|}{ Graduação } \\
\hline & $\begin{array}{l}\text { RP bruta (IC } \\
95 \%)\end{array}$ & $\mathbf{p}$ & $\begin{array}{l}\text { RP ajustada (IC } \\
95 \%)\end{array}$ & p \\
\hline Agenda não compartilhada com a EAB & $1,03(1,01-1,04)$ & 0,00 & $1,04(1,03-1,06)$ & 0,00 \\
\hline $\begin{array}{l}\text { Agenda não organizada para ofertar atividades de educação } \\
\text { em SB no território }\end{array}$ & $1,03(1,02-1,03)$ & 0,00 & $1,03(1,02-1,04)$ & 0,00 \\
\hline Agendamento das consultas em dias e horários específicos & $1,03(1,01-1,05)$ & 0,00 & $0,99(0,97-1,01)$ & 0,45 \\
\hline Sem garantia de retorno para continuidade de tratamento & $1,01(1,00-1,02)$ & 0,00 & $1,00(0,99-1,01)$ & 0,90 \\
\hline Não realização de Visitas Domiciliares & $1,08(1,07-1,10)$ & 0,00 & $1,08(1,07-1,09)$ & 0,00 \\
\hline $\begin{array}{l}\text { Não utilização de protocolo para definição de ações } \\
\text { prioritárias para VD }\end{array}$ & $1,07(1,05-1,08)$ & 0,00 & $1,06(1,04-1,07)$ & 0,00 \\
\hline
\end{tabular}

na formação profissional não se limite à qualificação puramente técnica ${ }^{13}$, garantida minimamente pelas universidades, pois a formação que pretende ser eficiente para o trabalho no SUS não pode esquecer a importância da comunidade na definição de suas necessidades, já que a prestação de atendimento somente tem sentido quando responde às demandas dos usuários do Sistema de Saúde ${ }^{14}$.

O trabalho em equipe multiprofissional, elemento central para trabalhar em SF, mostra-se deficiente em profissionais sem formação em SF, visto que muitos não compartilham a agenda de $\mathrm{SB}$ com os demais profissionais da $\mathrm{EAB}$. Isto corrobora com Teixeira ${ }^{15}$ e outros estudos ${ }^{16-18}$, contrapondo Silva e Trad. ${ }^{19}$, Baldani et al. ${ }^{20}$ e Vilarinho et al. ${ }^{21}$. Lourenço et al. ${ }^{22}$, em estudo que abrangeu 310 municípios que apresentavam ESB na ESF, verificou que $76,2 \%$ das ESB afirmaram existir integração com a EAB, porém esta não se manifestava no planejamento e/ou na troca de saberes. Desta forma, a abordagem integral dos indivíduos/famílias é facilitada pela soma dos olhares dos distintos profissionais que compõem as equipes interdisciplinares ${ }^{23}$, pois os problemas bucais não estão isolados de outros problemas que requerem a atuação integrada da $\mathrm{ESF}^{24}$. O trabalho em equipe, assim, é uma forma eficiente de estruturação, organização e de aproveitamento das habilidades humanas, possibilitando uma visão mais global e coletiva do trabalho, reforça o compartilhamento de tarefas e a necessidade de cooperação para alcançar objetivos comuns ${ }^{23,25}$. Sem esta interação com a EAB, as ações da ESB permanecem isoladas, reforçando a divisão do trabalho através de especialidades estanques que pouco interagem ${ }^{15}$ e limitando o processo de tra- balho à aplicação de estruturas rígidas do conhecimento técnico-estruturado ${ }^{23}$.

A Educação em Saúde, que desempenha papel essencial no novo modelo de atenção à saúde preconizado pela $\mathrm{PNAB}$, mostra-se pouco utilizada como ferramenta na produção do cuidado, uma vez que a maioria dos profissionais sem formação em SF não organizam sua agenda para ofertar atividades educativas em SB no território. Estes achados estão de acordo com Besen et al. ${ }^{13}$, Pimentel et al. ${ }^{16}$, Araújo e Dimenstein ${ }^{26}$, Cericato et al. ${ }^{17}$ e Pimentel et al. ${ }^{27}$, os quais identificam dificuldades de realização de atividades coletivas de educação em saúde pelos CD. Cabe destacar que a ESF tem como papel central uma prática educativa voltada para a Promoção de Saúde, como um conjunto de atividades orientadas a propiciar o melhoramento das condições de bem-estar e acesso a bens e a serviços sociais. Assim, dedicar um tempo e espaço para trabalhar questões que vão além do biológico com a população propulsionará o desenvolvimento de conhecimentos, atitudes e comportamentos favoráveis ao cuidado em saúde, através do processo de empoderamento e luta pelo alcance de estratégias que permitam um maior controle sobre suas condições de vida, individual e coletivamente ${ }^{13}$, garantindo uma atenção integral.

No que se refere ao agendamento de retorno daquele usuário que já iniciou tratamento, mais de $70 \%$ dos profissionais que não garantem este agendamento não têm formação em SF, o que evidencia a desconsideração dos atributos de acesso e longitudinalidade preconizados pela $\mathrm{AB}$. Evidencia-se também que, quanto à realização de tratamento concluído, a maioria dos profissionais que nunca realizam não dispõe de formação em 
SF. No cotidiano das EAB, a integralidade opera, desta forma, nos microprocessos de trabalho em saúde, nos fazeres de cada profissional que adota uma postura acolhedora e compromissada com os usuários, seus cuidados e a cura, ao estabelecer vínculo, condutas e prioridades equânimes para assisti-los em função de suas necessidades ${ }^{28}$. Em relação à SB, não facilitar o acesso aos usuários que já iniciaram tratamento, assim como não terminar o tratamento que foi proposto significa atuar ainda centrado na clínica e no enfoque biológico, em detrimento da dimensão integral que deve ter o cuidado. No entanto, não há estudos publicados que abordem essas dimensões do processo de trabalho - garantia de retorno e tratamento concluído - para que se pudesse comparar os resultados deste estudo com outros.

As VD, instrumentos centrais no processo de trabalho das ESF, as quais visam à produção de novos modos de cuidado ${ }^{29,30}$, não estão sendo realizadas por todos os profissionais das ESB, em especial por aqueles que possuem graduação somente. Segundo Araújo e Dimenstein ${ }^{26}$, Pimentel et al. ${ }^{27} \mathrm{e}$ Tomasi et al. ${ }^{31}$, as ESB ainda encontram dificuldades para a realização de VD, em contraponto ao encontrado por Sobrinho et al. ${ }^{32}$, Baldani et al. ${ }^{20} \mathrm{e}$ Lourenço et al. ${ }^{22}$. Segundo Giacomozzi e Lacerda ${ }^{33}$, a VD é uma forma de instrumentalizar os profissionais para o conhecimento da realidade de vida da população, bem como para estabelecer vínculos com a mesma. Desempenha papel estratégico na integralidade e humanização das ações, pois permite uma maior proximidade e responsabilização dos profissionais com as necessidades de saúde da população, de sua vida social e familiar ${ }^{29}$. Trata-se de uma tarefa difícil, não apenas pelo desgaste físico, mas, sobretudo, por ser altamente exigente do trabalho psíqui$\mathrm{Co}^{29}$; aponta a angústia e o sentimento de impotência e frustração diante de situações que põem em cheque o saber do profissional, uma realidade adversa que não se pode mudar, como a pobreza extrema e a violência que marcam a vida de muitas famílias ${ }^{34}$.

Além disso, não há a utilização de protocolo para definição de ações prioritárias nas VD, e esta utilização é menos frequente nos profissionais sem formação em SF. Ressalta-se que não há um critério estabelecido ou mesmo protocolo nacional para indicação de realização de VD, mas sim há alguns municípios que possuem protocolos locais. Dentre as propostas para sistematizar a $\mathrm{VD}$ na $\mathrm{AB}$, destacam-se aquelas focadas na estratificação de risco ou vulnerabilidade familiar para priorizar visitas a famílias de maior base/ risco social ${ }^{35}$. Lacerda et al. ${ }^{36}$ afirma que os profissionais demonstram que atuam e se organizam melhor quando há rotinas e protocolos que refletem sobre melhorias em suas práticas. Assim, a VD deve ser prestada de acordo com as características da comunidade atendida e dos recursos disponíveis para assistir pessoas com diferentes graus de incapacidade ${ }^{37}$.

Como limitações deste estudo, temos a fragmentação do processo de Avaliação Externa, que foi realizado por diferentes instituições e, assim, por diferentes equipes de avaliadores, potencial fonte de viés de aferição. Ainda, há o fato de nem todas as ESB serem de ESF (algumas, em pequeno número, fazem parte de UBS tradicionais parametrizadas - com critérios que as colocam em comparação com as ESB de SF), o que denota processos de trabalho que não contam com a realização de VD, por exemplo. Além disso, dos 4.272 profissionais entrevistados com formação em SF (34\%), somente 234 (1,9\%) possuem Residência em SF, considerada o padrão-ouro de formação para a SF, visto que permite uma vivência prática nos serviços, permeada por um suporte pedagógico específico e voltado para a necessidade da população $0^{38,39}$. Tal situação tornou impossível a utilização da Residência como uma categoria separada de análise.

É eminente, assim, a necessidade de qualificar os recursos humanos em SB para o SUS, pois é essencial superar os efeitos do ensino em odontologia, ainda curativista e individualista, que não se orienta pela situação epidemiológica, social, cultural e econômica da população. Continua sendo um desafio superar a fragmentação do ensino em saúde para que se possa potencializar a $\mathrm{AB}$ como porta de entrada preferencial do serviço, que coordena o cuidado do usuário, ao longo do tempo e de forma integral - garantindo ações de promoção, prevenção e recuperação da saúde.

Investir em formação, especificamente em SF, para atender às demandas do SUS, é de suma importância para a real mudança no modelo de atenção à SB, o qual ainda, muitas vezes, se encontra meramente travestido de novo, mas permanece atuando na mesma lógica que se perpetuou hegemônica por muitos anos. 


\section{Colaboradores}

NML Santos escreveu o artigo, o qual é oriundo de sua Dissertação de Mestrado, trabalhou na análise estatística e fez sua revisão. FN Hugo trabalhou na orientação do artigo, análise estatística e revisão.

\section{Referências}

1. Fernandes FM, Ribeiro JM, Moreira MR. Reflexões sobre avaliação de políticas de saúde no Brasil. Cad Saude Publica 2011; 27(9):1667-1677.

2. Almeida PF, Giovanella L. Avaliação em Atenção Básica à Saúde no Brasil: mapeamento e análise das pesquisas realizadas e/ou financiadas pelo Ministério da Saúde entre os anos de 2000 e 2006. Cad Saude Publica 2008; 24(8):1727-1742.

3. Brasil. Ministério da Saúde (MS). Política Nacional de Saúde Bucal. Brasília: MS; 2004.

4. Pinto HA, Sousa A, Ferla AA. O Programa Nacional de Melhoria do Acesso e da Qualidade da Atenção Básica: várias faces de uma política inovadora. Saúde debate 2014; 38(n. esp.):358-372.

5. Brasil. Ministério da Saúde (MS). Programa Nacional de Melhoria do Acesso e da Qualidade da Atenção Básica (PMAQ): manual Instrutivo. Brasília: MS; 2012.

6. Gil CRR. Formação de recursos humanos em saúde da família: paradoxos e perspectivas. Cad Saude Publica 2005; 21(2):490-498.

7. Batista KBC, Gonçalves OSJ. Education of Health Professionals for the SUS: meaning and care. Saúde Soc. 2011; 20(4):884-899.

8. Araújo D, Miranda MCG, Brasil SL. Formação de profissionais de saúde na perspectiva da integralidade. Rev. Baiana Saúde Pública 2007; 31(Supl. 1):20-31.

9. Guizardi FL, Stelet BP, Pinheiro R, Ceccim RB. A formação de profissionais orientada para a integralidade e as relações político-institucionais na saúde: uma discussão sobre a interação ensino-trabalho. In: Pinheiro R, Ceccim RB, Mattos RA, organizadores. Ensinar saúde: a integralidade e o SUS nos cursos de graduação na área de saúde. Rio de Janeiro: Abrasco; 2006. p. 153-177.

10. Garbin CAS, Sliba NA, Moimaz SAS, Santos KT. O papel das universidades na formação de profissionais na área de saúde. ABENO 2006; 6:6-10.

11. L'Abbate S. Educação e serviços de saúde: avaliando a capacitação dos profissionais. Cad Saude Publica 1999; 15(Supl. 2):15-27.

12. Seidl H, Vieira SP, Fausto MCR, Lima RCD, Gagno J. Gestão do trabalho na Atenção Básica em Saúde: uma análise a partir da perspectiva das equipes participantes do PMAQ-AB. Saúde debate 2014; 38(n. esp.):94-108.

13. Besen CB, Netto MS, Da Ros MA, Silva FWd, Silva CGd, Pires MF. A estratégia saúde da família como objeto de educação em saúde. Saúde Soc. 2007; 16(1):57-68.

14. Severino AJ. Educação e universidade: conhecimento e construção da cidadania. Interface (Botucatu) 2002; 6(10):117-124.

15. Teixeira MCB. A dimensão cuidadora do trabalho de equipe em saúde e sua contribuição para a odontologia. Cien Saude Colet 2006; 11(1):45-51.

16. Pimentel FC, Martelli PJL, Araújo Junior JLAC, Acioli RML, Macedo CLSV. Análise da atenção à saúde bucal na Estratégia de Saúde da Família do Distrito Sanitário VI, Recife (PE). Cien Saude Colet 2010; 15(4):21892196.

17. Cericato GO, Garbin D, Fernandes APS. A inserção do cirurgião-dentista no PSF: uma revisão crítica sobre as ações e os métodos de avaliação das Equipes de Saúde Bucal. RFO UPF 2007; 12(3):18-23. 
18. Pereira DQ, Pereira JCM, Assis MMA. A prática odontológica em Unidades Básicas de Saúde em Feira de Santana (BA) no processo de municipalização da saúde: individual, curativa, autônoma e tecnicista. Cien Saude Colet 2003; 8(2):599-609.

19. Silva IZQJ, Trad LAB. O trabalho em equipe no PSF: investigando a articulação técnica e a interação entre os profissionais. Interface (Botucatu) 2005; 9(16):25-38.

20. Baldani MH, Fadel CB, Possamai T, Queiroz MGS. A inclusão da odontologia no Programa Saúde da Família no Estado do Paraná, Brasil. Cad Saude Publica 2005; 21(4):1026-1035.

21. Vilarinho SMM, Mendes RF, Prado Júnior RR. Perfil dos cirurgiões-dentistas integrantes do programa saúde da família em Teresina (PI). Rev. odonto ciênc. 2007; 22(55):48-54.

22. Lourenço EC, Silva ACB, Meneghin MC, Pereira AC. A inserção de equipes de saúde bucal no Programa Saúde da Família no Estado de Minas Gerais. Cien Saude Colet 2009; 14(Supl. 1):1367-1377.

23. Araújo MBS, Rocha PM. Trabalho em equipe: um desafio para a consolidação da estratégia de saúde da família. Cien Saude Colet 2007; 12(2):455-464.

24. Faccin D, Sebold R, Carcereri DL. Processo de trabalho em saúde bucal: em busca de diferentes olhares para compreender e transformar a realidade. Cien Saude Colet 2010; 15(Supl. 1):1643-1652.

25. Motta PR. Desempenho em equipes de saúde: manual. Rio de Janeiro: Editora FGV; 2001.

26. Araújo YP, Dimenstein M. Estrutura e organização do trabalho do cirurgião-dentista no PSF de municípios do Rio Grande do Norte. Cien Saude Colet 2006; 11(1):219-227.

27. Pimentel FC, Martelli PJL, Araújo Junior JLAC, Lima AS, Santana VGD, Macedo CLSV. Evolução da assistência em saúde bucal na estratégia de saúde da família do município do Recife (PE) no período de 2001 a 2007. Rev. Baiana Saúde Pública 2008; 32(2):253-264.

28. Viegas SMF, Penna CMM. A construção da integralidade no trabalho cotidiano da equipe saúde da família. Esc. Anna Nery 2013; 17(1):133-141.

29. Cunha MS, Sá MC. A visita domiciliar na Estratégia de Saúde da Família: os desafios de se mover no território. Interface (Botucatu) 2013; 17(44):61-73.

30. Borges R, D’Oliveira AFPL. A visita médica domiciliar como espaço para interação e comunicação em Florianópolis, SC. Interface (Botucatu) 2011; 15(37):461-472.

31. Tomasi E, Facchini LA, Thumé E, Piccini RX, Osorio A, Silveira DS, Siqueira FV, Teixeira VA, Dilélio AS, Maia MFS. Características da utilização de serviços de Atenção Básica à Saúde nas regiões Sul e Nordeste do Brasil: diferenças por modelo de atenção. Cien Saude Colet 2011; 16(11):4395-4304.

32. Sobrinho JEL, Martelli PJL, Albuquerque MSV, Lyra TM, Farias SF. Acesso e qualidade: avaliação das Equipes de Saúde Bucal participantes do PMAQ-AB 2012 em Pernambuco. Saúde debate 2015; 39(104):136-146.

33. Giacomozzi CM, Lacerda MR. A prática da assistência domiciliar dos profissionais da estratégia de saúde da família. Texto Contexto Enferm 2006; 15(4):645-653.
34. Romanholi RMZ, Cyrino EG. A Visita domiciliar na formação de médicos: da concepção ao desafio do fazer. Interface (Botucatu) 2012; 16(42):693-705.

35. Savassi L, Carvalho H, Mariano F, Lamberti C, De Mendonça M, Yamana G, Pereira RPA. Proposta de protocolo de classificação de risco para o atendimento domiciliar individual na atenção primária. JMPHC 2013; 3(2):151-157.

36. Lacerda MR, Gomes IM, Kalinowski LC, Giacomozzi CM. Estratégias para avanços na prática do cuidado domiciliar. Cogitare Enferm 2010; 15(4):609-615.

37. Brasil. Ministério da Saúde (MS). Grupo Hospitalar Conceição. Lopes JMC, organizador. Manual de assistência domiciliar na atenção primária à saúde. Porto Alegre: Serviço de Saúde Comunitária do Grupo Hospitalar Conceição; 2003.

38. Nascimento DDG, Oliveira MAC. Competências profissionais e o processo de formação na residência multiprofissional em Saúde da Família. Saúde Soc. 2010; 19(4):814-827.

39. Nascimento DDG, Quevedo MP. Aprender fazendo: considerações sobre a Residência Multiprofissional em Saúde da Família na qualificação de profissionais da saúde. In: Bourget MMM, organizador. Estratégia Saúde da Família: a experiência da equipe de reabilitação. São Paulo: Martinari; 2008. p. 43-59.

Artigo apresentado em 24/05/2016

Aprovado em 13/12/2016

Versão final apresentada em 15/12/2016 
